\title{
Enseñanza de Física en Criminalística: Un Enfoque Práctico al Tema de Colisiones Inelásticas
}

\author{
José Augusto Bustamante Benitez, \\ Maestro en Ciencias en Física Educativa, \\ Instituto Politécnico Nacional, México \\ Mario Humberto Ramírez Díaz, \\ Doctor en Ciencias en Física Educativa, \\ Instituto Politécnico Nacional, México \\ Carlos Israel Aguirre Vélez, \\ Doctor en Ciencias en Tecnología Avanzada, \\ Instituto Politécnico Nacional, México
}

Doi:10.19044/esj.2019.v15n20p49 ～URL:http://dx.doi.org/10.19044/esj.2019.v15n20p49

\section{Resumen}

La Física es una de las ciencias más importantes en Criminología, que se puede utilizar para la explicación de una colisión vehicular hasta la reconstrucción de toda una escena delictiva. Este trabajo en particular trata de cómo se aplicó un sistema de estilos de aprendizaje basado en la estrategia de enseñanza 4MAT para alumnos de la licenciatura en Ciencias Forenses y Criminología. Para cada actividad de enseñanza propuesta se solicitó una prueba de aprendizaje, en la cual los estudiantes demostrarían su capacidad para comprender y resolver problemas. La aplicación de este sistema buscaba reducir los problemas que enfrentan los egresados en la aplicación de esta ciencia durante su vida profesional. Durante el desarrollo de la implementación de esta metodología se resolvieron varias carencias en habilidades manuales y estrategias de aprendizaje.

Palabras clave: Física Educativa, Ciencias Forenses, Estilos de Aprendizaje, Física para No-físicos 


\title{
Teaching Physics in Criminalistic Science: A Practical Approach to Theme of Inelastic Collisions
}

\author{
José Augusto Bustamante Benítez, \\ Maestro en Ciencias en Física Educativa, \\ Instituto Politécnico Nacional, México \\ Mario Humberto Ramírez Díaz, \\ Doctor en Ciencias en Física Educativa, \\ Instituto Politécnico Nacional, México \\ Carlos Israel Aguirre Vélez, \\ Doctor en Ciencias en Tecnología Avanzada, \\ Instituto Politécnico Nacional, México
}

\begin{abstract}
Physics is one of the most important sciences in Criminology, which can be used to explain a vehicular collision until the reconstruction of a whole criminal scene. This paper focuses on how a system of learning styles based on 4MAT system teaching strategy for undergraduate students in Forensic Sciences and Criminology was applied. For each proposed teaching activity, a learning test was requested through which the students would demonstrate their ability to understand and solve problems. The application of this system sought to reduce the problems faced by graduates in the application of this science in their lifetime profession. During the development of the implementation of this methodology, several shortcomings in manual skills and learning strategies were resolved.
\end{abstract}

Keywords: Physics Education, Forensic Science, Learning Styles, Physics for non-physicist

\section{Introducción}

En cualquier sistema de justicia penal los participantes deben ser profesionales que ayuden a los fiscales, jueces y abogados a culpar, sancionar y defender a las personas que participan en un hecho delictivo. Dichos profesionistas deben ser capaces de explicar la forma en la que ocurrió un hecho delictivo basándose en los métodos y técnicas propios de la Criminalística y las Ciencias Forenses. En este sentido, la Física auxilia a la Criminalística en la explicación de fenómenos como es el caso de aquellos que tienen que ver con el movimiento de los cuerpos y sus efectos; por ejemplo, la 
explicación del movimiento de proyectiles (balística forense) o la explicación de la colisión de vehículos (hechos debidos al tránsito terrestre).

Ya lo menciona García (2004):

"La base conceptual de la Ingeniería forense es la Física, ciencia teórica y experimental, cuyo objeto es investigar los fenómenos de la naturaleza. La Física define modelos abstractos (conceptos), expresados como relaciones matemáticas (ecuaciones, fórmulas), y verificadas a través de experimentos de laboratorio, que no sólo explican lo observado, sino que además pueden predecir nuevos fenómenos".

Un caso particular en relación a lo anterior es la colisión entre vehículos. La Física le ayuda al especialista en el área de Hechos de Tránsito Terrestre a interpretar y explicar los fenómenos que se involucran en la colisión. Por ello es fundamental el entendimiento de conceptos tales como: cantidades vectoriales y escalares, unidades de medida y su conversión, masa, energía-trabajo y su disipación, impulso, cantidad de movimiento, leyes de movimiento de Newton, tipos de colisiones, etc. Con estos conocimientos, el especialista puede narrar qué ocurre antes, durante y después de la colisión entre vehículos o entre un vehículo y el cuerpo de una persona, esto con la finalidad de realizar una reconstrucción que fortalecerá la explicación de las causas que dan origen a dicha colisión, para que así el causante de dicho hecho sea sancionado legalmente.

Una de las problemáticas de enseñar Física a los alumnos de programas en Criminalística radica en que la gran mayoría de dichos alumnos ingresan precisamente por considerarse a sí mismos no aptos para la Física ya que la relacionan con las Matemáticas. No obstante, en el plan de estudios de dichos programas se contempla la materia de principios básicos de Física y también se contemplan materias donde tendrán que aplicar lo aprendido en dicho curso (Como ejemplo http://www.cleu.edu.mx/CampusDFDos/licenciaturas.html).

Tomando en cuenta estos antecedentes, el objetivo de este trabajo es aplicar una estrategia educativa basada en los estilos de aprendizaje, específicamente en el Sistema 4MAT, pero con un enfoque en actividades prácticas o experimentales que ayudará a los alumnos de Ciencias Forenses y Criminalística a mejorar el aprendizaje sobre temas relacionados con las colisiones inelásticas.

\section{Antecedentes}

La Criminalística es una ciencia encargada del estudio del lugar donde ocurre un hecho delictivo con la finalidad de auxiliar al derecho penal en la explicación del cómo ocurrió el hecho. Esto se lleva a cabo analizando todos los factores que influyen en dicho hecho para así poder estar en la posibilidad 
de establecer quién fue el que cometió el delito y qué utilizó para lograr su cometido, lo cual ayuda al órgano jurisdiccional a sancionar dicha conducta.

Para Montiel (2003) la Criminalística es una "ciencia natural" ya que nace fundamentalmente de tres ciencias naturales: la Química, la Física y la Biología, por lo cual la define como: "una ciencia natural y penal, quemediante la aplicación de sus conocimientos, metodología y tecnología al estudio de las evidencias materiales asociativas, descubre y verifica de manera científica un hecho presuntamente delictuoso $\mathrm{y}$ al o a los presuntos autores $\mathrm{y}$ sus cómplices".

Por lo tanto, dentro de las ramas que prevén las Ciencias Forenses se encuentra obviamente la Física, ya que con el entendimiento de esta ciencia se pueden explicar los fenómenos que ocurren en delitos relacionados con el disparo de proyectiles (balística forense) por ejemplo, o delitos que ocurren como producto de la colisión de vehículos (Hechos de Tránsito Terrestre o Accidentología Vial), siendo estos últimosel tema principal de este estudio.

Para poder entender las colisiones, los estudiantes deben estar familiarizados con varios conceptos tales como: masa, cantidad escalar, cantidad vectorial, sistemas de unidades, velocidad, aceleración, fuerza, energía cinética, trabajo, impulso, cantidad de movimiento, principalmente.

A la falta de conocimiento de algunos principios de Física hay que agregar la falta de estrategias didácticas de los profesores que imparten dicha materia, ya que si bien la formación de dichos profesores está dentro del área de las ciencias Físico-Matemáticas, la mayoría de ellos no están relacionados con el ámbito forense, por lo que se apegan al currículo provisto por los programas universitarios para poder impartir la materia y no se enfocan en las necesidades de la Criminalística.

De igual manera hay que tomar en cuenta que los alumnos tienen problemas con esa estrategia de enseñanza, ya que se les dificulta en extremo la comprensión de los fenómenos que se analizan en la clase, esto debido a que su formación académica en el bachillerato, proviene de ramas relacionadas con las ciencias sociales y administrativas o medico biológicas (Medina \& Ramírez, 2019; Tacca, 2011).

La enseñanza de estos temas en general se sigue haciendo de una forma "tradicional" partiendo de la teoría hasta llegar a la resolución de problemas y en el camino entre una y otra, se realizan prácticas de laboratorio (Marušić \& Sliško, 2011; Torres, 2010).

Por todo lo anterior, es importante que los alumnos de Ciencias Forenses experimenten con los fenómenos físicos que se pueden presentar durante la comisión de un delito, ya que así podrán entender y explicar con mayor facilidad dichos fenómenos.

La experimentación acerca a los alumnos a una nueva forma de ver las ciencias naturales, ya que mediante ella podrán analizar fenómenos físicos y 
les facilitará la comprensión de los modelos matemáticos que explican dichos fenómenos. Así mismo les permitirá, en caso de ser necesario, realizar modelos para explicar otro tipo de fenómenos que durante su vida profesional se encuentren.

En este trabajo se mostrarán los resultados de una propuesta didáctica educativa basada en el Sistema 4MAT de estilos de aprendizaje con un enfoque hacia la experimentación, derivada de una evaluación diagnóstica y dirigida a atender a estudiantes de Ciencias Forenses y Criminalística, tomando como caso particular el tema de colisiones en temas de incidentes de tránsito.

\section{II.1 Sistema 4MAT experimental}

La Física es una ciencia basada en fenómenos tangibles que se interpretan con modelos teóricos en un lenguaje matemático. Los profesores de esta ciencia, en general, hacen más énfasis en la resolución de ejercicios con vistas a que los estudiantes aprueben los exámenes que en la enseñanza experimental de la Física. Sin embargo, el componente experimental en el aprendizaje de la Física es fundamental e imprescindible en la comprensión de este conocimiento (Rodríguez-Llerena \& Llovera-González, 2014; RuízMendoza \& Ramírez, 2015).

Por otro lado, existen diferentes modelos y esquemas de estilos de aprendizaje como los planteados por Kolb (1984) y el de McCarthy (2006). En este último, McCarthy retoma el trabajo de Kolb añadiendo el procesamiento de los hemisferios cerebrales para establecer un ciclo de instrucción de 8 pasos, el Sistema 4MAT. En este sistema cada estilo de aprendizaje está definido por la forma en que los estudiantes aprenden.

El proceso contínuo del sistema 4MAT se mueve desde la reflexión a la acción, la combinación de estas dos posibles elecciones en el individuo forma las diferencias individuales, a las cuales llama Estilo 1, Estilo 2, Estilo 3 y Estilo 4. Para el Sistema 4MAT los estilos de aprendizaje describen comportamientos generales y por lo tanto un estudiante no puede ser identificado con un único estilo. Sin embargo, se pueden identificar características generales para cada estilo de aprendizaje, las cuales se muestran a continuación:

- Estilo 1. Obtienen de la enseñanza un valor personal. Disfrutan las discusiones en pequeños grupos que nutren la conversación.

- Estilo 2. Guardan la verdad. Requieren exactitud y orden. Se sienten cómodos con las reglas y construyen la realidad a partir de éstas. Son exigentes en la forma de expresión; metódicos y precisos.

- Estilo 3. Se lanzan a la acción; pretenden que lo aprendido les sea útil y aplicable. No aceptan que les proporcionen las respuestas antes de explorar todas las posibles soluciones. 
- Estilo 4. Descubren las cosas por sí mismos. Tienen una fuerte necesidad de experimentar libertad en su aprendizaje, y tienden a transformar cualquier cosa.

Un aspecto importante en el Sistema 4MAT es el desarrollo del ciclo de aprendizaje que permite la realización de actividades dirigidas a incentivar a cada uno de los estilos de aprendizaje de forma que, al terminar un ciclo, los estudiantes de todos los estilos se vean beneficiados al desarrollar actividades dirigidas no sólo a su estilo preponderante, sino que además potencializarán los estilos menos preferidos en su forma de percibir y procesar la información.

Este tipo de ciclos de aprendizaje han sido probados, en el caso particular de aprendizaje de la Física, con éxito en los últimos años y con temas específicos. Ejemplos de lo anterior lo representan las pruebas hechas en temas como mecánica (Ramírez, 2010; Artamonova, Ramírez \& Mosquera, 2014), termodinámica (Najera, 2015) o resistencia de materiales (Rosado \& Guzmán, 2012). Sin embargo, en todas las propuestas anteriores se considera al trabajo experimental como dirigido únicamente a atender al estilo 3 de aprendizaje.

Las actividades experimentales (AE) en Física se pueden agrupar en 4 tipos (Aguirre \& Ramírez, 2017):

1) Análisis de video,

2) Demostración experimental,

3) Trabajo experimental fuera del ambiente escolar, y

4) Análisis y solución de un caso práctico.

Cada tipo de actividad promueve el desarrollo de ciertas competencias por parte de los estudiantes y tienen diferentes características en su implementación.

\section{Metodología \\ III. 1 Muestra}

Esta investigación se realizó en el Colegio Libre de Estudios Universitarios, Campus Ciudad de México, en donde se imparte la Licenciatura en Criminalística y Criminología, las maestrías en Criminalística y una maestría en Criminología, así como especialidades que tienen que ver con el ramo de las Ciencias Forenses.

La licenciatura tiene una duración de nueve cuatrimestres. En el primer cuatrimestre se les imparte la materia de Principios Básicos de Física, debido a la relación que esta ciencia tiene con el desarrollo de otras materias como son Acústica Forense, que se imparte en el quinto cuatrimestre, Hechos de Tránsito Terrestre, que se imparte en el octavo cuatrimestre y Balística, que se 
imparte en el noveno cuatrimestre (El plan de estudios se encuentra disponible en https://goo.gl/WJEDny).

El grupo quedó conformado por 25 alumnos de los cuales cuatro alumnos eran de octavo cuatrimestre, en donde cursan la materia de Hechos de Tránsito Terrestre, cuatro alumnos de quinto cuatrimestre, donde cursan la materia de Acústica Forense, y diecisiete alumnos de segundo cuatrimestre, los cuales un cuatrimestre anterior cursaron la materia de Principios Básicos de Física.

\section{III.2 Evaluación diagnóstica}

Se aplicó una evaluación diagnóstica compuesta por tres secciones; en la primera se les pide a los alumnos desarrollar algunos conceptos relacionados con el movimiento de los cuerpos; en la segunda sección se les pide a los alumnos completar algunos razonamientos relacionados con el movimiento de los cuerpos y en la tercer sección se proponen preguntas relacionadas con la aplicación de la Física en la Criminalística.

Con la aplicación del diagnóstico se pudieron detectar los aciertos y ciertas carencias que los alumnos tenían en cuanto a la conceptualización de ciertos fenómenos. A continuación, se presenta un análisis detallado de los resultados de cada sección de la evaluación diagnostica.

\begin{tabular}{|c|l|}
\hline Preguntas de diagnóstico & \multicolumn{1}{|c|}{ Resultado } \\
\hline ¿Qué entiende por materia? & $\begin{array}{l}\text { De los 25 integrantes del grupo, 14 respondieron la pregunta } \\
\text { utilizando conceptos y definiciones acordes a los } \\
\text { presentados en libros de texto; así mismo utilizaron } \\
\text { ejemplos que se aplicaban al concepto de masa.4 de los } \\
\text { alumnos utilizaron conceptos que no se acercaban a los } \\
\text { descritos en alguna bibliografía, además de que no utilizaron } \\
\text { ningún medio para ejemplificar el fenómeno. 2 de los } \\
\text { integrantes del grupo utilizaron ideas propias para explicar }\end{array}$ \\
$\begin{array}{l}\text { la pregunta o utilizaron solamente un ejemplo de dicha } \\
\text { propiedad. Solamente 5 de los alumnos no respondieron a la } \\
\text { pregunta. }\end{array}$ \\
\hline ¿Qué entiende por masa? & $\begin{array}{l}\text { De los 25 integrantes del grupo, 6 respondieron la pregunta } \\
\text { utilizando conceptos y definiciones acordes a los presentados } \\
\text { en libros de texto, así mismo utilizaron ejemplos que se } \\
\text { aplicaban al concepto de masa. 10 de los alumnos utilizaron } \\
\text { conceptos que no se acercaban a los descritos en alguna } \\
\text { bibliografía, además de que no utilizaron ningún medio para } \\
\text { ejemplificar el fenómeno. 7 de los integrantes del grupo } \\
\text { utilizaron ideas propias para explicar la pregunta o utilizaron } \\
\text { solamente un ejemplo de dicha propiedad. Solamente 2 de los } \\
\text { alumnos no respondieron a la pregunta. }\end{array}$ \\
\hline Qué entiende por movimiento? & $\begin{array}{l}\text { De los 25 integrantes del grupo, 14 respondieron la pregunta } \\
\text { utilizando conceptos y definiciones acordes a los presentados } \\
\text { en libros de texto; así mismo utilizaron ejemplos que se }\end{array}$ \\
\hline
\end{tabular}




\begin{tabular}{|c|c|}
\hline & $\begin{array}{l}\text { aplicaban al concepto de masa. } 5 \text { de los alumnos utilizaron } \\
\text { conceptos que no se acercaban a los descritos en alguna } \\
\text { bibliografía, además de que no utilizaron ningún medio para } \\
\text { ejemplificar el fenómeno. } 4 \text { de los integrantes del grupo } \\
\text { utilizaron ideas propias para explicar la pregunta o utilizaron } \\
\text { solamente un ejemplo de dicha propiedad. Solamente } 2 \text { de los } \\
\text { alumnos no respondieron a la pregunta. }\end{array}$ \\
\hline ¿Qué entiende por posición? & $\begin{array}{l}\text { De los } 25 \text { integrantes del grupo, } 12 \text { respondieron la pregunta } \\
\text { utilizando conceptos y definiciones acordes a los presentados } \\
\text { en libros de texto; así mismo utilizaron ejemplos que se } \\
\text { aplicaban al concepto de masa. } 7 \text { de los alumnos utilizaron } \\
\text { conceptos que no se acercaban a los descritos en alguna } \\
\text { bibliografía, además de que no utilizaron ningún medio para } \\
\text { ejemplificar el fenómeno. } 2 \text { de los integrantes del grupo } \\
\text { utilizaron ideas propias para explicar la pregunta o utilizaron } \\
\text { solamente un ejemplo de dicha propiedad y solamente } 4 \text { de } \\
\text { los alumnos no respondieron a la pregunta. }\end{array}$ \\
\hline ¿Qué entiende por cantidad escalar? & $\begin{array}{l}\text { De los } 25 \text { integrantes del grupo, ningún alumno respondió la } \\
\text { pregunta utilizando conceptos y definiciones acordes a los } \\
\text { presentados en libros de texto; así mismo utilizaron ejemplos } \\
\text { que se aplicaban al concepto de masa. } 8 \text { de los alumnos } \\
\text { utilizaron conceptos que no se acercaban a los descritos en } \\
\text { alguna bibliografía, además de que no utilizaron ningún } \\
\text { medio para ejemplificar el fenómeno. } 2 \text { de los integrantes del } \\
\text { grupo utilizaron ideas propias para explicar la pregunta o } \\
\text { utilizaron solamente un ejemplo de dicha propiedad y } \\
\text { solamente } 15 \text { de los alumnos no respondieron a la pregunta. }\end{array}$ \\
\hline ¿Qué entiende por cantidad vectorial? & $\begin{array}{l}\text { De los } 25 \text { integrantes del grupo, } 1 \text { alumno respondió la } \\
\text { pregunta utilizando conceptos y definiciones acordes a los } \\
\text { presentados en libros de texto; así mismo utilizaron ejemplos } \\
\text { que se aplicaban al concepto de masa. } 7 \text { de los alumnos } \\
\text { utilizaron conceptos que no se acercaban a los descritos en } \\
\text { alguna bibliografía, además de que no utilizaron ningún } \\
\text { medio para ejemplificar el fenómeno. } 2 \text { de los integrantes del } \\
\text { grupo utilizaron ideas propias para explicar la pregunta o } \\
\text { utilizaron solamente un ejemplo de dicha propiedad y } \\
\text { solamente } 15 \text { de los alumnos no respondieron a la pregunta. }\end{array}$ \\
\hline ¿Qué entiende por velocidad? & $\begin{array}{l}\text { De los } 25 \text { integrantes del grupo, } 8 \text { respondieron la pregunta } \\
\text { utilizando conceptos y definiciones acordes a los presentados } \\
\text { en libros de texto; así mismo utilizaron ejemplos que se } \\
\text { aplicaban al concepto de masa. } 11 \text { de los alumnos utilizaron } \\
\text { conceptos que no se acercaban a los descritos en alguna } \\
\text { bibliografía, además de que no utilizaron ningún medio para } \\
\text { ejemplificar el fenómeno. } 2 \text { de los integrantes del grupo } \\
\text { utilizaron ideas propias para explicar la pregunta o utilizaron } \\
\text { solamente un ejemplo de dicha propiedad. Solamente } 4 \text { de los } \\
\text { alumnos no respondieron a la pregunta. }\end{array}$ \\
\hline ¿Qué entiende por aceleración? & $\begin{array}{l}\text { De los } 25 \text { integrantes del grupo, } 6 \text { respondieron la pregunta } \\
\text { utilizando conceptos y definiciones acordes a los presentados } \\
\text { en libros de texto; así mismo utilizaron ejemplos que se }\end{array}$ \\
\hline
\end{tabular}




\begin{tabular}{|c|c|}
\hline & $\begin{array}{l}\text { aplicaban al concepto de masa. } 14 \text { de los alumnos utilizaron } \\
\text { conceptos que no se acercaban a los descritos en alguna } \\
\text { bibliografía, además de que no utilizaron ningún medio para } \\
\text { ejemplificar el fenómeno. } 3 \text { de los integrantes del grupo } \\
\text { utilizaron ideas propias para explicar la pregunta o utilizaron } \\
\text { solamente un ejemplo de dicha propiedad. Solamente } 2 \text { de los } \\
\text { alumnos no respondieron a la pregunta. }\end{array}$ \\
\hline $\begin{array}{l}\text { ¿Qué tipo de cantidad son la velocidad y la } \\
\text { aceleración? }\end{array}$ & $\begin{array}{l}\text { De los } 25 \text { integrantes del grupo, } 2 \text { respondieron la pregunta } \\
\text { utilizando conceptos y definiciones acordes a los presentados } \\
\text { en libros de texto; así mismo utilizaron ejemplos que se } \\
\text { aplicaban al concepto de masa. } 8 \text { de los alumnos utilizaron } \\
\text { conceptos que no se acercaban a los descritos en alguna } \\
\text { bibliografía, además de que no utilizaron ningún medio para } \\
\text { ejemplificar el fenómeno. } 2 \text { de los integrantes del grupo } \\
\text { utilizaron ideas propias para explicar la pregunta o utilizaron } \\
\text { solamente un ejemplo de dicha propiedad. Solamente } 13 \text { de } \\
\text { los alumnos no respondieron a la pregunta. }\end{array}$ \\
\hline ¿Qué entiende por energía? & $\begin{array}{l}\text { De los } 25 \text { integrantes del grupo, } 8 \text { respondieron la pregunta } \\
\text { utilizando conceptos y definiciones acordes a los } \\
\text { presentados en libros de texto; así mismo utilizaron } \\
\text { ejemplos que se aplicaban al concepto de masa. } 8 \text { de los } \\
\text { alumnos utilizaron conceptos que no se acercaban a los } \\
\text { descritos en alguna bibliografía, además de que no utilizaron } \\
\text { ningún medio para ejemplificar el fenómeno. } 4 \text { de los } \\
\text { integrantes del grupo utilizaron ideas propias para explicar } \\
\text { la pregunta o utilizaron solamente un ejemplo de dicha } \\
\text { propiedad y solamente } 5 \text { de los alumnos no respondieron a } \\
\text { la pregunta. }\end{array}$ \\
\hline ¿Qué entiende por deformación? & $\begin{array}{l}\text { De los } 25 \text { integrantes del grupo, } 15 \text { respondieron la pregunta } \\
\text { utilizando conceptos y definiciones acordes a los presentados } \\
\text { en libros de texto; así mismo utilizaron ejemplos que se } \\
\text { aplicaban al concepto de masa. } 2 \text { de los alumnos utilizaron } \\
\text { conceptos que no se acercaban a los descritos en alguna } \\
\text { bibliografía, además de que no utilizaron ningún medio para } \\
\text { ejemplificar el fenómeno. } 3 \text { de los integrantes del grupo } \\
\text { utilizaron ideas propias para explicar la pregunta o utilizaron } \\
\text { solamente un ejemplo de dicha propiedad y solamente } 5 \text { de } \\
\text { los alumnos no respondieron a la pregunta. }\end{array}$ \\
\hline ¿Qué tipos de colisiones conoce? & $\begin{array}{l}\text { De los } 25 \text { integrantes del grupo, } 2 \text { respondieron la pregunta } \\
\text { utilizando conceptos y definiciones acordes a los presentados } \\
\text { en libros de texto; así mismo utilizaron ejemplos que se } \\
\text { aplicaban al concepto de masa. } 2 \text { de los alumnos utilizaron } \\
\text { conceptos que no se acercaban a los descritos en alguna } \\
\text { bibliografía, además de que no utilizaron ningún medio para } \\
\text { ejemplificar el fenómeno. } 11 \text { de los integrantes del grupo } \\
\text { utilizaron ideas propias para explicar la pregunta o utilizaron } \\
\text { solamente un ejemplo de dicha propiedad y solamente } 19 \text { de } \\
\text { los alumnos no respondieron a la pregunta. }\end{array}$ \\
\hline
\end{tabular}




\begin{tabular}{|l|l|l|}
\hline \multicolumn{1}{|c|}{ Razonamiento } & \multicolumn{1}{|c|}{ Opciones } & \multicolumn{1}{c|}{ Resultado } \\
\hline 1. A mayor velocidad, mayor: & $\begin{array}{l}\text { a. energía. } \\
\text { b. aceleración. } \\
\text { c. masa. }\end{array}$ & $\begin{array}{l}\text { De 25 alumnos 10 contestaron } \\
\text { correctamente, 15 contestaron } \\
\text { incorrectamente }\end{array}$ \\
\hline 2. A mayor energía, mayor: & $\begin{array}{l}\text { a. deformación. } \\
\text { b. materia. } \\
\text { c. aceleración. }\end{array}$ & $\begin{array}{l}\text { De los 25 alumnos, 6 } \\
\text { contestaron correctamente, 19 } \\
\text { contestaron incorrectamente. }\end{array}$ \\
\hline $\begin{array}{l}\text { 3. En un cuerpo que se } \\
\text { mueve, la velocidad seguirá: } \\
\text { a. el mismo sentido que el } \\
\text { movimiento. } \\
\text { b. un sentido contrario al } \\
\text { movimiento. } \\
\text { c. ninguna de las anteriores. }\end{array}$ & $\begin{array}{l}\text { De los 25 alumnos, 16 } \\
\text { contestaron correctamente, } \\
\text { contestaron correctamente. }\end{array}$ \\
\hline $\begin{array}{l}\text { 4. Las unidades de velocidad } \\
\text { están expresadas en: }\end{array}$ & $\begin{array}{l}\text { a. unidades de longitud sobre } \\
\text { unidades de tiempo al } \\
\text { cuadrado. } \\
\text { b. unidades de longitud } \\
\text { sobre unidades de tiempo. } \\
\text { c. unidades de área sobre } \\
\text { unidades de tiempo. }\end{array}$ & $\begin{array}{l}\text { De los 25 alumnos 11 } \\
\text { contestaron correctamente, 14 } \\
\text { contestaron incorrectamente. }\end{array}$ \\
\hline $\begin{array}{l}\text { 5. Si un vehículo recorre 45 } \\
\text { kilómetros cada hora, avanza: }\end{array}$ & $\begin{array}{l}\text { b. 10.5 metros cada segundo. } \\
\text { c. 12.5 metros cada segundo. } \\
\text { segundo. }\end{array}$ & $\begin{array}{l}\text { De los 25 alumnos 19 } \\
\text { contestaron correctamente, 6 } \\
\text { contestaron incorrectamente. }\end{array}$ \\
\hline
\end{tabular}

\begin{tabular}{|c|l|}
\hline Pregunta & \multicolumn{1}{|c|}{ Resultado. } \\
\hline $\begin{array}{c}\text { ¿Cuál es la relación de la física con la } \\
\text { Criminalística? }\end{array}$ & $\begin{array}{l}\text { Los alumnos tienen clara esta relación y la basan en } \\
\text { que la Física es un auxiliar de la Criminalística y la } \\
\text { ayuda a explicar fenómenos donde interviene algún } \\
\text { cuerpo en movimiento e incluso cuando tienen que } \\
\text { emplear instrumentos de medición. }\end{array}$ \\
\hline $\begin{array}{c}\text { Durante su vida profesional ¿Cuál será } \\
\text { la relevancia de realizar el análisis y } \\
\text { explicación de fenómenos físicos? }\end{array}$ & $\begin{array}{l}\text { Los alumnos tienen presente que al estar ejerciendo } \\
\text { tendrán que explicar fenómenos relacionados con } \\
\text { choques o movimiento de proyectiles ante un juez; sin } \\
\text { embargo, están temerosos en ese aspecto ya que no se } \\
\text { sienten preparados para hacerlo. }\end{array}$ \\
\hline $\begin{array}{c}\text { Qué fenómenos físicos tienen mayor } \\
\text { relación con su licenciatura? }\end{array}$ & $\begin{array}{l}\text { Los alumnos relacionan el movimiento de cuerpos y } \\
\text { proyectiles, además de lo relacionado con acústica } \\
\text { forense, y fenómenos como la vibración. }\end{array}$ \\
\hline $\begin{array}{c}\text { ¿Cuál ha sido su experiencia con el } \\
\text { aprendizaje de la Física y su aplicación } \\
\text { en la Criminalística? }\end{array}$ & $\begin{array}{l}\text { Su experiencia la define como difícil ya que aún no } \\
\text { entienden cómo aplicaran muchas cosas y ven muy } \\
\text { lejano que puedan ejercer en delitos que involucren la } \\
\text { explicación de la física, ya que sienten que para ello } \\
\text { hay que ser ingenieros o físicos. }\end{array}$ \\
\hline \multicolumn{2}{|c}{}
\end{tabular}


Con base en este análisis se hizo la construcción del ciclo de aprendizaje, considerando las AE y las EA que permitierán superar las carencias y errores conceptuales que manifestaron los estudiantes.

\section{Resultados}

\section{IV.1 Construcción de estrategia propuesta}

Basados en los resultados de la evaluación diagnóstica se diseñaron 4 actividades experimentales (AE) y sus correspondientes evidencias de aprendizaje (EA) relacionados con la colisión de vehículos.

Las $\mathrm{AE}$ se relacionaron con la reproducción de fenómenos físicos que se encuentran presentes en la comisión de delitos cometidos en la conducción de vehículos. Con estas actividades se pretende lograr que los alumnos puedan desarrollar argumentos que utilicen como base para la explicación de dichos fenómenos ante un juez y tomar la decisión de absolver o sancionar este tipo de acciones.

Debido a que las AE son diversas, se tiene la necesidad de proponer EA que den cuenta sobre el trabajo realizado por parte de los estudiantes fuera del laboratorio. Las EA consideradas en este trabajo fueron cuatro: 1) discusiones grupales, 2) exposición oral, 3) reporte escrito, y 4) Cartel (poster o infograma) y presentación ante grupo.

Todo el ciclo de aprendizaje (Figura 1) se aplica durante seis sesiones de dos horas durante dos semanas. Para poder evaluar el trabajo realizado durante las sesiones, se construyeron instrumentos de evaluación para cada actividad.

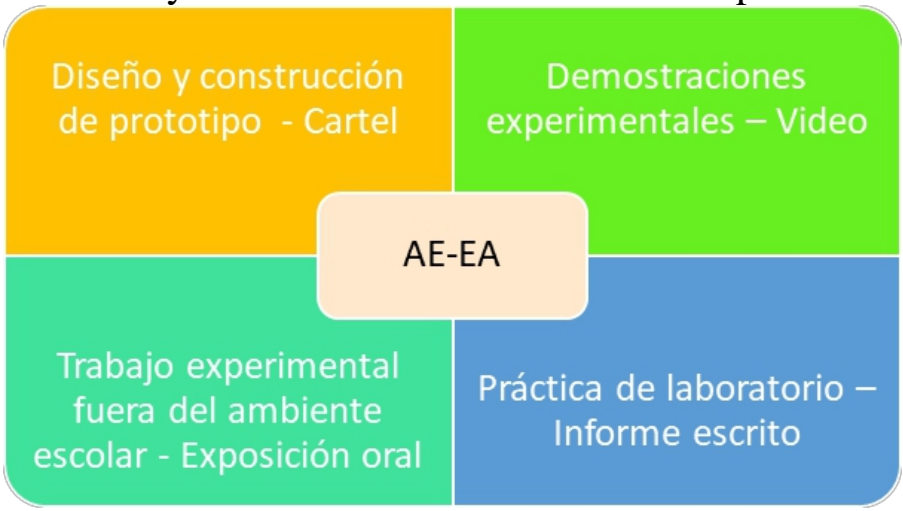

Figura 1. Ciclo de aprendizaje con Actividades Experimentales y evidencias para los 4 estilos del 4MAT

\section{IV.2 Discusión grupal}

El objetivo de la $\mathrm{AE}$ proyección de video es que los alumnos realicen observaciones referentes a establecer una relación entre la velocidad de los vehículos y la deformación que se causa debida a la colisión. Para tener la 
evidencia de esta actividad, se le solicita a los alumnos que realicen un reporte de lo observado y de sus deducciones.

La discusión grupal se lleva a cabo de la siguiente manera:

1. Se proyecta un video en el que se observa una colisión vehicular (figura 2) donde un automóvil es impactado en contra de una barrera fija. En dicho video se observa la forma en la que la colisión afecta al vehículo, deformándolo y reduciendo su energía cinética; también se observan los efectos que la colisión causa sobre un "dommie" que se encuentra en el asiento del conductor.

2. Se forman equipos de trabajo con la finalidad de que cada equipo responda un cuestionario de 5 preguntas, relacionado con lo observado en el video. Estas preguntas se diseñaron para que los alumnos discutan los fenómenos físicos que se presentan durante una colisión como son la deformación de los materiales, aceleración, velocidad, energía, inercia, trabajo, etc.

3. Se realiza una discusión grupal para explicar los fenómenos antes mencionados y en qué efectos de la colisión intervienen los conceptos físicos.

4. Con la intención de retroalimentar la discusión, el profesor realiza una breve exposición acerca de los conceptos para así facilitar el entendimiento de dichos fenómenos y que de esta manera puedan explicarlos con mayor fluidez y puedan identificarlos en el momento de analizar una colisión.

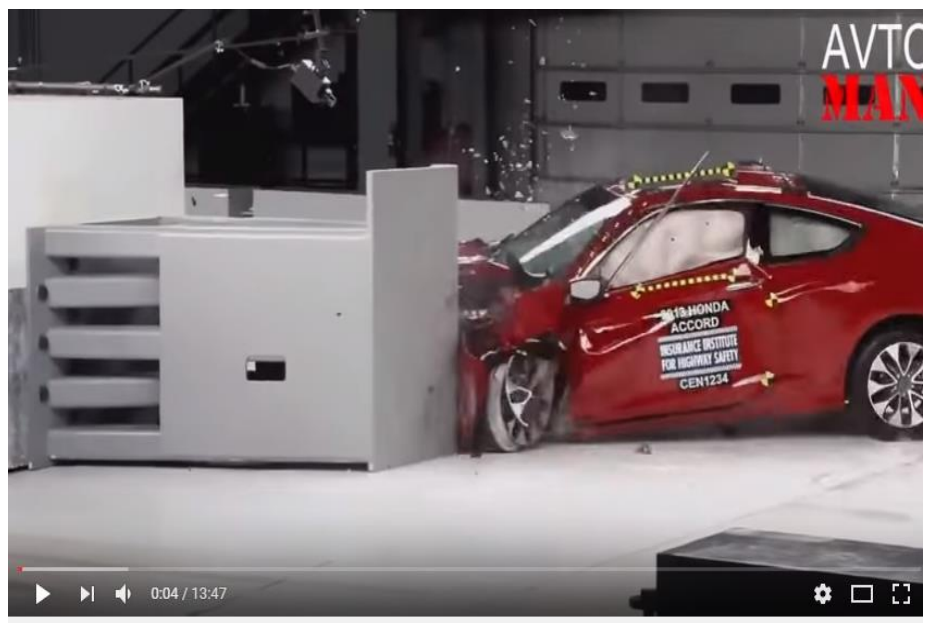

Mirra!! Autos puestos a prueba en choques, parte 2

Figura 2. Muestra del video usado en la AE. 


\section{IV.3 Demostración experimental}

En la AE Demostración experimental, se tiene como objetivo reproducir el fenómeno de colisiones con base al modelo explicado y realizar pruebas tomando nota de lo observado. La AE se lleva a cabo con los siguientes pasos:

1. Un artefacto desarrollado previamente (figura 3) se usa para demostrar la relación que existe entre velocidad y deformación. Este artefacto consiste en un estructura de madera que sujeta láminas de aluminio y un péndulo, con la finalidad de que mediante las características del péndulo, se pueda controlar la velocidad del cuerpo que colisionaría en contra de una lámina y así poder analizar la relación existente entre la velocidad del cuerpo y la deformación que se produce en la lámina; es decir, se debe establecer una relación basada en el aumento de velocidad y si dicho aumento provoca una mayor deformación en la lámina.

2. Los alumnos realizan pruebas modificando la masa que colisiona en contra de la lámina para determinar si, considerando las mismas velocidades tomadas en cuenta para la primer parte del experimento, existe una diferencia en la deformación, pero ahora debida al cambio en la masa. Para este experimento, los alumnos además de colisionar la lámina tienen que tomar datos referentes al péndulo en el cual se encontraba sujeta la masa que colisiona; dichos datos les ayudarán a calcular la velocidad a la que se moverá dicho cuerpo. Esas características están relacionadas con la amplitud del péndulo, la velocidad angular, el largo de la cuerda de la cual cuelga el cuerpo colisionador. Se les proporcionan además las ecuaciones necesarias para con esos datos poder calcular la velocidad del cuerpo en metros sobre segundo.

3. Los estudiantes realizan un reporte en el cual describen y explican la demostración. Dentro del reporte también se encuentra una tabla con los datos que el alumno obtiene para establecer una relación que modele los fenómenos que observa.

4. Los alumnos realizan una comparación con la realidad mediante fotografías de la medición de deformaciones en vehículos colisionados.

5. Se presentan los modelos matemáticos que se utilizan para calcular la velocidad de un vehículo tomando como base la deformación que provoca. 


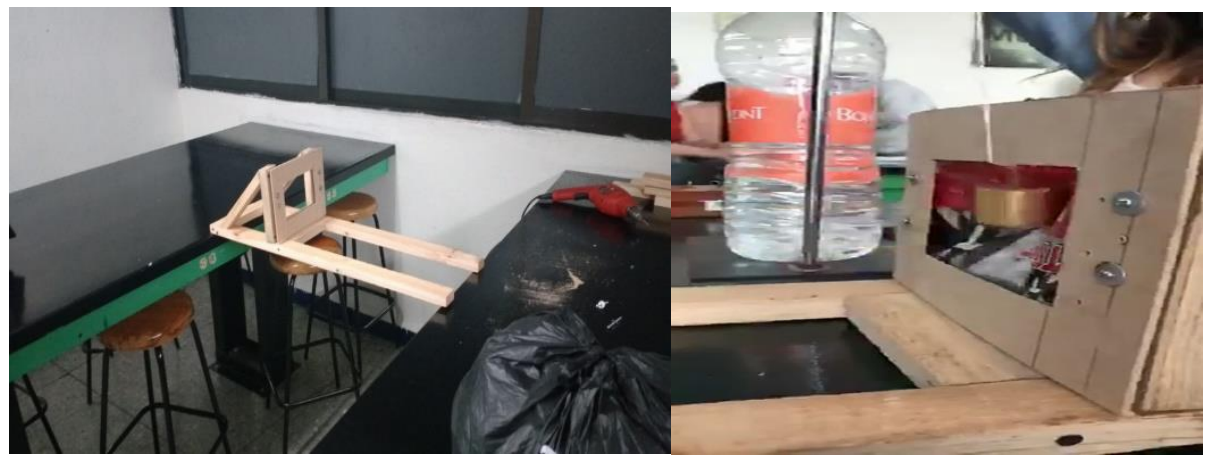

Figura 3. Prototipo utilizado en la AE Demostración experimental.

Los instrumentos de evaluación no pueden estar en contra de la normatividad de la institución de aplicación. En el caso de esta actividad se entrega un formato de reporte de práctica "Tradicional", la cual se evalúa con el instrumento 1 (Instrumento 1) que se muestra a continuación:

Instrumento 1. Instrumento para evaluar la práctica en AE Demostración experimental.

\begin{tabular}{|c|c|c|c|c|}
\hline Criterio & Indicadores & Valor & $\begin{array}{l}\text { Puntaje } \\
\text { obtenido }\end{array}$ & Observaciones \\
\hline $\begin{array}{l}\text { Durante la } \\
\text { explicación }\end{array}$ & $\begin{array}{l}\text { Los alumnos centran su atención en la } \\
\text { explicación que se da de cómo se llevará a } \\
\text { cabo la practicidad y de los fenómenos que se } \\
\text { deben analizar para poder medirlos y } \\
\text { explicarlos. }\end{array}$ & $20 \%$ & & \\
\hline $\begin{array}{l}\text { Durante la } \\
\text { realización }\end{array}$ & $\begin{array}{l}\text { Los integrantes del equipo reproducen el } \\
\text { modelo explicado y realizan pruebas, tomando } \\
\text { nota de los fenómenos que se observan y } \\
\text { poniendo especial énfasis en aquellos que se } \\
\text { les pidió analizar. }\end{array}$ & $20 \%$ & & \\
\hline $\begin{array}{c}\text { Trabajo en } \\
\text { equipo }\end{array}$ & $\begin{array}{l}\text { El equipo se distribuye las tareas que debe } \\
\text { realizar, ningún integrante queda sin una } \\
\text { actividad, intercambian dichas tareas con la } \\
\text { finalidad de que todos realicen por lo menos } \\
\text { una vez cada actividad. }\end{array}$ & $25 \%$ & & \\
\hline $\begin{array}{l}\text { Contenido } \\
\text { del reporte }\end{array}$ & $\begin{array}{l}\text { El alumno realiza el llenado de cada uno de los } \\
\text { apartados que incluye el formato que se les } \\
\text { proporcionó para realizar el reporte de la } \\
\text { práctica, explica los fenómenos que pudo } \\
\text { observar y realiza la narración de los mismos } \\
\text { utilizando un lenguaje técnico adecuado; } \\
\text { además incluye una conclusión de lo } \\
\text { observado. }\end{array}$ & $15 \%$ & & \\
\hline & & $100 \%$ & & \\
\hline
\end{tabular}




\section{IV.4 Trabajo experimental fuera de clase}

Para esta AE, se elige un experimento en el que el alumno pueda observar las trayectorias que siguen los cuerpos después de una colisión. El primer objetivo de este experimento es que los alumnos comprendan los fenómenos relacionados con la conservación del momento lineal, el cual es de utilidad cuando necesitan determinar las variables relacionadas con las colisiones entre vehículos cuando estas ocurren en un cruce o intersección. Como segundo objetivo está el que el estudiante comprenda las cantidades vectoriales involucradas en fenómenos de colisiones y cómo éstas dependen de factores tales como la masa de los cuerpos y el ángulo en que ocurre la colisión.

La AE se describe a continuación:

1. Los alumnos van a un billar, donde realizan la experimentación. Con la ayuda de las bolas de dicho juego e instrumentos de medición se determina la relación entre el centro geométrico de las bolas a colisionar y la distancia que alcanzan después del golpe con el taco.La finalidad del experimento es que los alumnos encuentren la relación que tiene la posición en la que se da el golpe y el ángulo de salida de las bolas, lo cual tiene que ver con la trayectoria después de la colisión.

2. Los datos obtenidos son registrados en una tabla que se encuentra en el formato de práctica que los alumnos deberán presentar con la finalidad de explicar dichos resultados ante el grupo.

3. Durante la puesta en marcha de este experimento se les solicita a los alumnos tomar imágenes fotográficas, así como grabaciones en video que les sirvan como referencia para realizar una exposición oral de lo realizado durante el experimento.

\section{IV.5 Análisis de un caso práctico y presentación ante grupo}

Los estudiantes realizan una investigación en medios impresos y electrónicos con el objetivo de encontrar información relacionada con una colisión entre vehículos y realizar un cartel en el que el tema central sea la explicación de los fenómenos físicos que se involucran en la colisión que documentaron. Posteriormente, y como parte de la evidencia de aprendizaje, además del cartel, los alumnos hacen una presentación oral de lo investigado, explicando al grupo la forma en la que se desarrolló el hecho vehicular (Figura 4). 

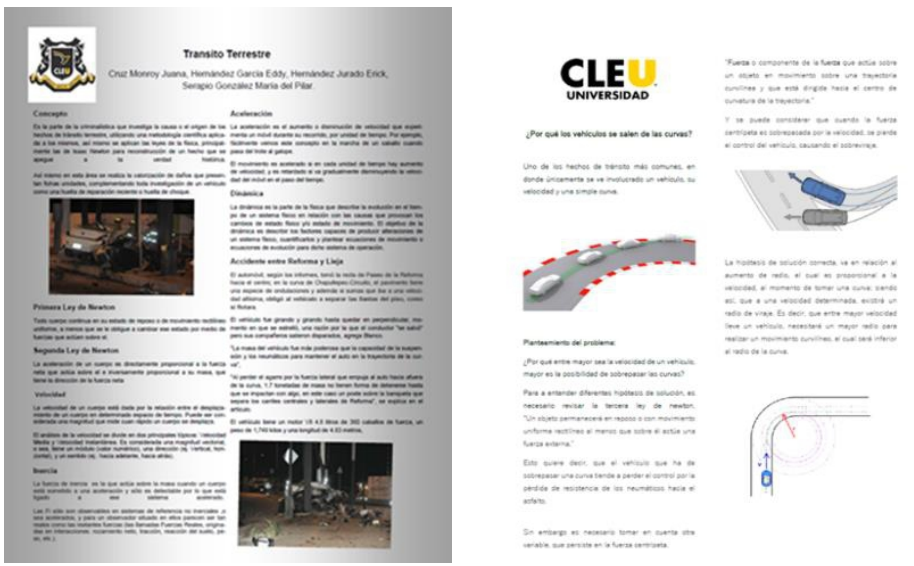

Figura 4. Ejemplo de carteles realizados por los estudiantes en la AE.

Para la AE Análisis de un caso práctico y presentación ante grupo se tiene un instrumento de evaluación (Instrumento 2) dirigido a hacer del conocimiento de los estudiantes los criterios de dicha evaluación, el cual se presenta a continuación:

Instrumento 2. Instrumento de evaluación dirigido a la AE Análisis de un caso práctico y presentación ante grupo.

\begin{tabular}{|c|l|c|c|c|}
\hline Criterio & \multicolumn{1}{|c|}{ Indicadores } & Valor & $\begin{array}{c}\text { Puntaje } \\
\text { obtenido }\end{array}$ & Observaciones \\
\hline Trabajo en & $\begin{array}{l}\text { Al inicio de su presentación emiten } \\
\text { una atractiva introducción al tema, } \\
\text { incluyendo los datos de } \\
\text { identificación. Cubren todos los } \\
\text { temas a profundidad emitiendo } \\
\text { algunos ejemplos. Identifican los } \\
\text { detalles de la información más } \\
\text { importantes, ubicándolos en las } \\
\text { categorías adecuadas. Todos los } \\
\text { integrantes conocen el tema } \\
\text { completamente y son capaces de } \\
\text { explicar lo realizado. }\end{array}$ & $\mathbf{2 0 \%}$ & & \\
\hline Desarrollo del & $\begin{array}{l}\text { En la presentación se puede } \\
\text { observar que los alumnos siguieron } \\
\text { las indicaciones del experimento, } \\
\text { además se apoyaron en } \\
\text { instrumentos de medición para } \\
\text { tener una mayor presión a la hora } \\
\text { de tomar datos. }\end{array}$ & $\mathbf{1 0 \%}$ & & \\
experimento & & & \\
\hline
\end{tabular}




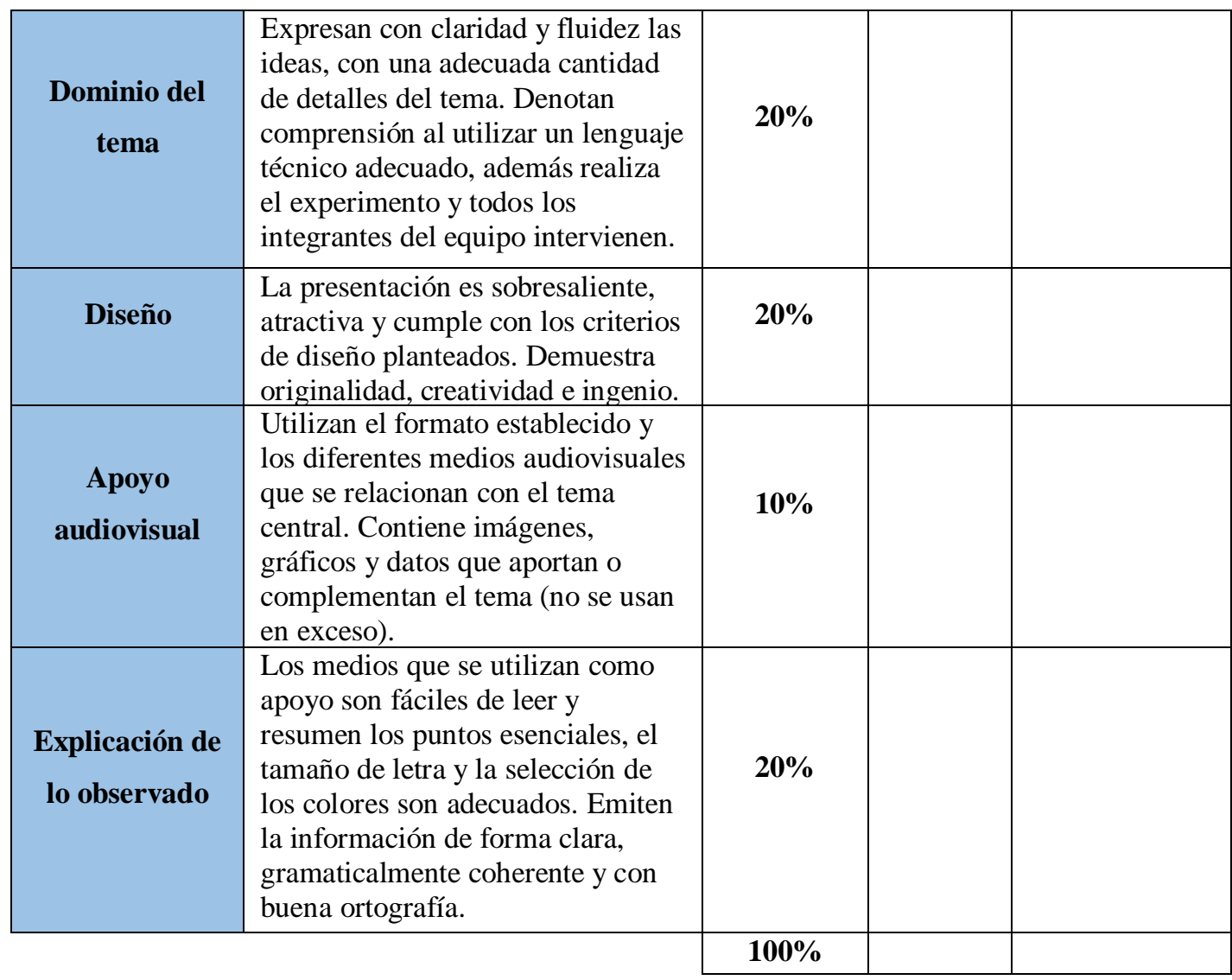

\section{Conclusiones}

La importancia de aplicar una nueva estrategia de enseñanza con los alumnos de la licenciatura en Criminalística o aquellas relacionadas con las ciencias forenses radica en que no todos aprendemos de la misma forma, además de que las necesidades que los alumnos de estas licenciaturas tienen comparadas con las que están relacionadas con las ramas físico-matemáticas son totalmente diferentes ya que los primeros tienen la necesidad de hablar de contenidos aplicados directamente a un delito. Tomando en cuenta esto se propuso implementar el Sistema 4MAT práctico.

De acuerdo con los resultados obtenidos a partir del instrumento diagnóstico se hacen evidentes deficiencias en los estudiantes acerca de temas básicos de física como masa, velocidad, aceleración, cantidades vectoriales y escalares. Además, no es clara para los estudiantes la relación de las cantidades físicas con casos prácticos de la vida real. No obstante, manifiestan que es importante el conocimiento de la física en la criminalística, por lo que se hace necesario mejorar las estrategias de aprendizaje orientadas específicamente a casos prácticos de aplicación en ciencias criminalísticas. 
Por tal motivo, en este trabajo se buscó proponer una estrategia que pudiera atender esta necesidad de formación, tomando como un primer caso de estudio el tema de colisiones inelásticas.

La propuesta se basó en el Sistema 4MAT con un enfoque práctico (Aguirre \& Ramírez, 2017) ya que este permite introducir AE dirigidas específicamente a atender las necesidades de formación particulares de estudiantes de ciencias criminalísticas. En el caso de la propuesta se buscó propiciar aprendizaje conceptual en temas relacionados con las colisiones inelásticas, sin entrar en conflicto con las normas institucionales de la escuela de implementación, por lo que se plantean EA como reportes de práctica, presentaciones orales, construcción de carteles y el fortalecimiento en los trabajos en equipo, para en todo momento relacionarlas con las ciencias forenses y la explicación y reconstrucción de hechos delictivos.

Con la propuesta que se presenta se espera que los estudiantes refuercen no solamente los conocimientos de física requeridos en criminalística desde una óptica práctica de aplicación, sino además habilidades como el trabajo en equipo, el autoaprendizaje y la aplicación de conocimientos a la realidad.

\section{References:}

1. Artamonova, I., Ramírez, M. \& Mosquera, J. (2014). Resultados cuantitativos de la aplicación del Sistema 4MAT en Mecánica en la Universidad del Quindio. Latin American Journal of Physics Education, 8(4) 4511-1-8.

2. García, A. (2004). La ingeniería Forense Su aplicación a la investigación de los siniestros viales. Recuperado de http://www.perarg.com.ar/docs/asist-Let/documentos/2-1003Ingenieria-Forense-y-siniestros-viales.pdf

3. Kolb, D. (1984). Experiential Learning: Experience as the source of Learning and Development. Englewood cliffs, New Jersey: Prentice Hall.

4. Marušić, M. \& Sliško, J. (2011). Influence of Three Different Methods of Teaching Physics on the Gain in Students' Development of Reasoning. International. Journal of Science Education, 34(2), 301326.

5. McCarthy, B. \& McCarthy, D. (2006). Teaching Around 4MAT Cycle: Designing Instruction for Di-verse Learners With Diverse Learning Styles. Thousand Oaks, California, Corwin Press.

6. Montiel Sosa, J. (2003). Criminalística Tomo 1. México: Limusa.

7. Ramírez, M. (2010). Aplicación del sistema 4MAT en la enseñanza de la Física a Nivel Universitario. Revista Mexicana de Física E, 56(1), 29-40. 
8. Najera, C. \& Ramírez, M. (2015). Estudio del grado de motivación para el aprendizaje de principios de la termodinámica utilizando el Sistema 4MAT de estilos de aprendizaje. Revista de Enseñanza de la Física, 27 (2), 7-18.

9. Rodríguez-Llerena, D. \& Llovera-González, J. (2014). Estrategias de enseñanza en el laboratorio docente de Física para estudiantes de ingeniería. Latin A.merican Journal of Physics Education, 8(4), 1-8.

10. Rosado, C. \& Guzmán, D. (2012). The 4MAT system applied to a blended learning scenario. Latin American Journal of Physics Education. 6 Suppl. I, 275-279.

11. Ruíz-Mendoza, J. \& Ramírez, M. (2015). Vinculo de la teoría con la práctica para la comprensión de la Óptica Geométrica en el Nivel Superior en las escuelas de Ingeniería de la UANL a partir del Modelo por Competencias. Caderno Brasileiro de Ensino de Física, 32 (2), 498-516.

12. Tacca, D. (2011). La enseñanza de las ciencias naturales en la educación básica. Investigación Educativa, 14(6), 139-152.

13. Torres, M. (2010). La enseñanza tradicional de las ciencias versus las nuevas tendencias educativas. Revista electrónica Educare, 14(10), $1409-42-58$. 